\title{
A search for very young Planetary Nebulae
}

\author{
G. Umana ${ }^{1}$, L. Cerrigone ${ }^{2}$, C. Trigilio ${ }^{1}$, and R. A. Zappalà ${ }^{2}$ \\ 1 Istituto di Radioastronomia del CNR, Stazione VLBI di Noto, CP 141 Noto, Italy \\ e-mail: g.umana@ira.cnr.it \\ 2 Dipartimento di Fisica e Astronomia, Università di Catania, Via S. Sofia 78, 95123 Catania, Italy
}

Received 8 April 2003 / Accepted 23 July 2004

\begin{abstract}
Despite numerous efforts, the transition from Asymptotic Giant Branch (AGB) stars to Planetary Nebulae (PN) is a poorly understood phase of stellar evolution. We have therefore carried out interferometric (VLA) radio observations of a sample of hot post-AGB stars, selected on the basis of their optical and infrared properties. Ten sources out of the 16 observed were detected. This indicates that most of our targets are surrounded by a nebula where the ionization has already started. This definitively determines the evolutionary status of the selected sources and provides us with a unique sample of very young Planetary Nebulae (yPNe). A comparison with another sample of yPNe confirms our working hypothesis that our targets are indeed very young, probably just in the transition toward PN. Finding transition objects is extremely important as they can provide unique clues for a better understanding of this important phase of stellar evolution.
\end{abstract}

Key words. stars: AGB and post-AGB - planetary nebulae: general - radio continuum: stars

\section{Introduction}

The formation and early evolution of Planetary Nebulae (PNe) is a quite obscure phase of stellar evolution. Recently HST has provided high-quality images of PNe which quite often reveal jets or bipolar structures whose origin it is difficult to explain. In particular, it is quite challenging to understand how the spherically symmetric Asymptotic Giant Branch (AGB) circumstellar shells transform themselves into the extremely asymmetric envelopes observed in evolved PNe.

Recent models propose different agents in shaping PNe such as high-speed, collimated outflows or jets that operate during the early post-AGB evolutionary phase (Sahai \& Trauger 1998; Lee \& Sahai 2003).

Very recently, high sensitivity, high resolution MERLIN observations of IRAS 18276-1431 in the OH maser lines 1612 and $1667 \mathrm{MHz}$ have made it possible, for the first time, to detect and measure the circumstellar magnetic field in a PPN (Bains et al. 2003). This result suggests that the magnetic field may play a fundamental role in the process that shapes the circumstellar envelopes in the post-AGB evolutionary phase.

During the last few years many observational programs have been devoted to recognizing new planetary and protoplanetary nebulae among unidentified IRAS sources with far infrared colors similar to those of known planetary nebulae (Pottasch et al. 1988; van de Steene \& Pottasch 1993; Garcia-Lario et al. 1997a). The final aim of these studies is to fully understand the process of formation of Planetary Nebulae through the discovery and analysis of new transition objects in the short phase between the end of the AGB and the onset of the ionization in the nebula.
The number of known transition objects is extremely small, tens compared to more than a thousand PNe identified in our Galaxy (Kwok 1994). This is related to the short evolutionary timescales involved (a few thousand years).

As post AGB stars/proto-PNe evolve into early stages of Planetary Nebulae, part of the shell begins to be ionized by the central star. The post-AGB objects detected by IRAS extended from non variable $\mathrm{OH}-\mathrm{IR}$ stars to $\mathrm{M}, \mathrm{K}, \mathrm{G}, \mathrm{F}$, A supergiant types. This sequence appears to represent the evolution of the post-AGB stars towards hotter spectral types and we should also expect to find some B-type stars among the hottest postAGB stars. There is a small sub-group of B-type stars, called $\mathrm{BQ}\left[\mathrm{]}\right.$ stars, defined as $B_{\mathrm{e}}$ with forbidden emission lines, and Parthasarathy \& Pottasch (1989), on the basis of their IR excess, recognized them as potential candidates to be new transition objects.

$\mathrm{BQ}$ [ ] stars, however, are not a well defined group, and there is still a controversy on their evolutionary stage. Parthasarathy $\&$ Pottasch (1989) suggested that at least a fraction of them could be hot post-AGB stars in an early stage of their evolution as proto-PNe. The common characteristics of these kinds of star are:

- numerous permitted and forbidden emission lines of several elements, in addition to the absorption lines typical of a B1 I-II star;

- underabundance of carbon and metals;

- high galactic latitude;

- presence of PN-type detached cold circumstellar shells.

These characteristics are typical for low-mass, highly evolved post-AGB stars. 
Table 1. Programme stars.

\begin{tabular}{|c|c|c|c|c|c|c|c|c|c|c|}
\hline Name & IRAS & $\alpha_{\text {J2000 }}^{\alpha_{(1)}}$ & $\begin{array}{c}\delta_{\text {J2000 }} \\
\text { (1) }\end{array}$ & $\alpha_{\text {radio }}$ & $\overline{\delta_{\text {radio }}}$ & $\bar{V}$. & S.T. & $\begin{array}{c}F_{3.6 \mathrm{~cm}} \\
\mathrm{mJy}\end{array}$ & $\begin{array}{c}\sigma \\
\mathrm{mJy}\end{array}$ & $\begin{array}{l}\mathrm{rms} \\
\mathrm{mJy}\end{array}$ \\
\hline OY Gem & $06556+1623$ & 065830.3 & +161925 & 065830.3 & +161926.2 & $11.5^{(2)}$ & $B Q^{(2)}$ & 0.55 & 0.03 & 0.02 \\
\hline \multirow[t]{2}{*}{ Hen 3-1347 } & 17074-1845 & 171024.1 & -184901 & & & $9.5^{(4)}$ & $B 3 I I I e^{(4)}$ & & & 0.03 \\
\hline & $17203-1534$ & 172311.8 & -153715 & & & $12.5^{(4)}$ & $B I I I e^{(4)}$ & & & 0.03 \\
\hline LS 4331 & 17381-1616 & 174059.9 & -161758 & 174100.1 & -161812 & $12.2^{(4)}$ & $B 1 I e^{(4)}$ & 1.42 & 0.05 & 0.03 \\
\hline Hen 3-1475 & $17423-1755$ & 174514.2 & -175647 & 174514.1 & -175645 & $11.0^{(2)}$ & $B e^{(2)}$ & 0.26 & 0.03 & 0.03 \\
\hline SAO 209306 & $17460-3114$ & 174916.5 & -311518 & 174916.5 & -311518 & $7.9^{(4)}$ & $B 0 I I e^{(4)}$ & 1.29 & 0.05 & 0.04 \\
\hline V886 Her & $18062+2410$ & 180820.1 & +241043 & 180820.1 & +241043 & $11.6^{(4)}$ & $B 1 I I I e^{(4)}$ & 1.46 & 0.05 & 0.03 \\
\hline LS 63 & 18371-3159 & 184021.6 & -315649 & 184022.0 & -315649 & $11.9^{(4)}$ & $B 1 I e^{(4)}$ & 0.62 & 0.03 & 0.03 \\
\hline \multirow[t]{2}{*}{ LS 5112} & $18379-1707$ & 184048.5 & -170436 & & & $11.8^{(4)}$ & $B 1 I I I e^{(4)}$ & & & 0.03 \\
\hline & $18435-0052$ & 184606.9 & -004855 & & & $11.0^{(4)}$ & $B 2 I I^{(4)}$ & & & 0.03 \\
\hline BD -11 4747 & $18442-1144$ & 184704.8 & -114102 & 184704.0 & -114112 & $9.3^{(2)}$ & $B e^{(2)}$ & 19.21 & 0.60 & 0.05 \\
\hline \multirow[t]{2}{*}{ LS IV -02 29} & $19157-0247$ & 191822.5 & -024209 & & & $11.5^{(4)}$ & $B 1 I I I^{(4)}$ & & & 0.03 \\
\hline & $19336-0400$ & 193617.5 & -035324 & 193617.5 & -035325 & $12.5^{(4)}$ & $B 1 I e^{(4)}$ & 9.74 & 0.29 & 0.03 \\
\hline LS II +23 17 & $19399+2312$ & 194205.5 & +231859 & & & $10.4^{(4)}$ & $B 1 I I I^{(4)}$ & & & 0.03 \\
\hline LS IV -12 111 & 19590-1249 & 200149.8 & -124117 & 200149.8 & -124117 & $11.3^{(4)}$ & $B 1 I e^{(4)}$ & 2.76 & 0.08 & 0.03 \\
\hline LS II +34 26 & $20462+3416$ & 204816.6 & +342724 & 204816.6 & +342724 & $11.1^{(3)}$ & $B I e^{(3)}$ & 0.42 & 0.03 & 0.03 \\
\hline
\end{tabular}

(1) Optical coordinates are FK5 2000.0 as reported by SIMBAD database.

(2) Parthasarathy (1993a).

(3) Parthasarathy (1993b).

(4) Parthasarathy et al. (2000a).

In some cases the central star may just have become hot enough to photoionize the circumstellar envelope ejected during the previous AGB phase, as the presence of low excitation nebular emission lines in the spectrum seems to indicate.

\section{Observations and results}

\subsection{Sample selection}

In order to find new very young Planetary Nebulae we have selected a sample of 16 hot post-AGB stars from the most recent compilations, namely Parthasarathy (1993a); Conlon et al. (1993) and Parthasarathy et al. (2000a). All the selected candidates have high galactic latitude, infrared excess, spectral type B1 I-II and nebular emission lines in their spectrum (Table 1). In particular the last two requirements maximize the possibility of detecting a radio nebula. Moreover in one of the proposed targets, namely SAO 85766, the strong variations observed in the spectrum suggest that it has rapidly evolved in the last 50 years (Parthasarathy et al. 2000b) and its evolutionary state appears to be similar to that observed in the case of the hot post-AGB star SAO 244567, where a radio nebula has been detected (Umana et al. 2004).

The detection of radio nebulae associated to the selected targets would definitively establish the evolutionary status of this kind of objects, producing a unique sample of very young PNe.

Successive higher resolution radio observations of the detected targets will allow us to study nebular morphologies in the very early phases of evolution of PNe and thus clarify at what stage of the AGB-PN evolution those asymmetries are established, providing strong clues to the process shaping PNe.

\subsection{The VLA data}

The observations were carried out using the $\mathrm{VLA}^{1}$ in two different runs, the first on June 8, 2001, from 06:06 UT to 11:33 UT, and the second on June 10, 2001 from 18:56 UT to $20: 24 \mathrm{UT}$.

We observed our targets at $8.4 \mathrm{GHz}$ (X-Band), using two independent $50 \mathrm{MHz}$ bands. The choice of the observing frequency is a compromise between the sensitivity of the instrument and the higher probability of observing in the optically thin region of the spectra. The array was in $\mathrm{CnB}$ configuration, providing a typical beam size of $\sim 2.5^{\prime \prime}$.

A typical observing cycle consisted of 10 min integration time, preceded and followed by a 2-min observation of the phase calibrator. This basic sequence was repeated at least 3 times in order to improve the signal to noise ratio and thus to ensure the necessary sensitivity for the detection of weak sources. The flux density scale was determined by observing 3C286 and 3C48, while phase calibration was obtained from observations of phase calibrators close to our targets.

The data processing was performed using the standard programs of the NRAO Astronomical Image Processing System (AIPS). To achieve the highest possible signal to noise ratio, the mapping process was performed using the natural weighting and the dirty map was CLEANed down as close as possible to the theoretical noise. To estimate the noise level in the maps we analyzed an area on the map, whose dimension is of the order of more than $100 \theta_{\text {syn }}^{2}$, away from the phase center and free from evident radio sources. Its consistency with the expected theoretical noise was also checked.

\footnotetext{
The Very Large Array is a facility of the National Radio Astronomy Observatory which is operated by Associated Universities, Inc. under cooperative agreement with the National Science Foundation.
} 
Of 16 observed sources, we detected a total of ten. The source position and the flux density determination were obtained by fitting a Gaussian brightness distribution (JMFIT). The uncertainty in the source position is a function of the rms in the map, the resolution $\left(\theta_{\mathrm{syn}}\right)$, and the peak flux density of the source in the image $\left(F_{\text {peak }}\right)$, namely:

$\sigma_{\text {pos }} \sim \frac{\operatorname{rms} \theta_{\text {syn }}}{2 F_{\text {peak }}} \sim \frac{\theta_{\text {syn }}}{2 S / N}$

where $S / N$ is the signal to noise ratio for the source detection. For the worst case in our sample we derive a $\sigma_{\text {pos }}$ of about 0 o' $^{\prime} 12$.

Our results are summarized in Table 1, where the radio flux density, with associated error $(\sigma)$ and the rms of the map, are reported. The error associated to the flux density estimation is a combination of the rms in the map plus the amplitude calibration error. Therefore, $\sigma$ is derived from:

$\sigma=\sqrt{(\mathrm{rms})^{2}+\left(\sigma_{\mathrm{cal}} F_{3.6 \mathrm{~cm}}\right)^{2}}$

where $\sigma_{\text {cal }}$ is the error associated to the flux calibration, which is of the order of $2-3 \%$, and $F_{3.6 \mathrm{~cm}}$ is the measured flux density. At $\mathrm{CnB}$ resolution all the detected targets are point-like or slightly resolved radio sources, i.e. $\theta_{\text {source }} \leq 2^{\prime \prime}$.

All the sources in our sample were checked for $20 \mathrm{~cm}$ emission using the NRAO VLA Sky Survey (NVSS, Condon et al., 1998). Only two of our detected targets were present in the survey, namely IRAS 18442 and IRAS 19336, with fluxes at $1.4 \mathrm{GHz}$ of $8.4 \pm 0.6$ and $8.8 \pm 0.6 \mathrm{mJy}$ respectively.

It is not easy to verify whether the non-detections are related to a larger distance or due to an intrinsic characteristic of the source such as the evolution of the ionization structure. In fact, distances to planetary nebulae are notoriously very difficult to be estimate.

Traditionally, distances are derived through statistical methods, which are based on a particular characteristic of the nebula, assumed to be invariant. For example, the Shklovsky method assumes that all PN have the same ionized masses (Cahn \& Kaler 1971). Since this is often not the case, statistical methods are considered not to be reliable (Terzian 1993).

Recently, Gauba et al. (2003) modelled the near and farinfrared flux distribution of a small sample of hot post-AGB candidates, with IRAS colors similar to those of PNe. Their sample includes four of our targets, namely IRAS 17074+1845, IRAS 17423-1755, IRAS 19157-0247 and IRAS 19399+2312. Using the DUSTY code (Ivezic et al. 1999), the authors modelled the circumstellar shells of their sample and derived various physical parameters, including an estimate of the distance. They derived a distance of 3.7, 3.7, 0.9 and $0.7 \mathrm{Kpc}$ respectively to IRAS $17074+1845$, IRAS $17423-1755$ IRAS 19157-0247 and IRAS 19399+2312, with an uncertainty of about $10 \%$ for each source, depending on the assumed core mass.

Their results indicate, at least for a small fraction of our sample, that the lack of detectable radio flux should be related to intrinsic factor and not to the distance. As a matter of fact, the undetected sources IRAS 19157-0247 and IRAS 19399+2312 have distances much smaller than the detected source IRAS 17423-1755.
We stress again the difficulties in deriving distances to PNe, regardless of the used method, as it will lead to uncertainties by a typical factor of 2 or 3 . As an example, the distance to IRAS 17423-1755 has been derived by different authors, using different methods, to range from $8.3 \mathrm{kpc}$ (Borkowski \& Harrington 2001) to $5.4 \mathrm{kpc}$ (Riera et al. 2003). Therefore, results presented by Gauba et al. (2003) provide only an indication and should be taken with caution.

\section{Notes on individual sources}

To point out any characteristics common to the detected sources, besides selection criteria, we summarize, in the following, the physical properties of each source as derived from the literature. When available, a previous radio measurement is reported and compared with our result. All the targets in our sample are associated witn an IRAS source. When a discrepancy between radio, optical and IRAS coordinates is present, a comment is added at the end of each source section.

\section{IRAS $06556+1623$}

When it was discovered, in 1932, this star showed some P Cygni line profiles in its spectrum. Ciatti et al. (1974) pointed out the presence, in the spectrum, of several strong emission lines. The continuum emission was well represented by a $\mathrm{B} 0.5$ star and the line ratios for [O I], [O II], [O III] were not compatible with a unique set of density and temperature of the nebula. This suggests that the star is surrounded by an extended gaseous shell composed of regions of different temperatures, densities and velocities (Klutz \& Swings 1977).

By comparing their spectrophotometric data with other data previously reported by other authors, Arkhipova \& Ipatov (1982) concluded that the volume and density of the gaseous shell had been increasing for the last 40 years. The more recent spectra are those obtained by Jaschek et al. (1996), which were collected between 1990 and 1993. Many lines are present, including several classic nebular lines such as [O III]. No significant differences with previous similar work are reported. Attempts to detect radio emission were made by Sistla \& Seung (1975) at $8.4 \mathrm{GHz}$ and by Altenhoff et al. (1976) at $10.7 \mathrm{GHz}$, but both studies the sensitivity was not high enoigh to detect the source, the rms being of the order of $5 \mathrm{mJy}$.

\section{IRAS 17381-1616}

The star was included in a sample of PN candidates selected from the IRAS Point Source Catalog on the basis of their far-IR colours (Preite-Martinez 1988). IRAS 17381-1616 was included in a list of objects that were probably not PN but PN-related. Parthasarathy et al. (2000a) classified it as a B1Ibe star, with $\mathrm{H} \beta$ and $\mathrm{H} \gamma$ emission lines, suggesting that the object was a post-AGB star.

The interferometer was pointed at LS 4331, which is believed to be the optical counterpart of IRAS 17381-1616. However, we did not find the radio source at the expected optical coordinates, but we detected a $1.42 \pm 0.05 \mathrm{mJy}$ radio source at a radio position of $\alpha=17: 41: 00.05$ and $\delta=-16: 18: 12.45$, corresponding to a shift of $2.16^{\prime \prime}$ in right ascension and about $16^{\prime \prime}$ in declination, or about $6.5 \theta_{\text {syn }}$. 
However the radio positions are shifted by only $\Delta \alpha=2$ '. 16 and $\Delta \delta=1^{\prime \prime}$. 15 with respect to the IRAS coordinates, well inside the IRAS ellipse error $\left(34^{\prime \prime} \times 8^{\prime \prime}\right)$. We can thus conclude that our radio source is associated to the IRAS source.

\section{IRAS 17423-1755}

The source was believed to be a massive population I B-type star, but Bobrowsky et al. (1995) recognized it as a new planetary nebula.

High resolution spectroscopy and optical images reveal a complex bipolar morphology: a dense central region (disk) from which large-scale flows collimate into bipolar jets (Bobrowsky et al. 1995; Riera et al. 1995; Borkowski \& Harrington 2001).

Parthasarathy et al. (2000a) found Balmer lines having a P Cygni profile, blue-shifted by $-400 \mathrm{~km} \mathrm{~s}^{-1}$ and from the analysis of $\mathrm{H}_{\alpha}$ profiles Sanchez Contreras \& Sahai (2001) pointed out the presence of two different winds, one of these probably associated to the post-AGB outflow prior to its interaction with the AGB wind.

Knapp et al. (1995) detected it at $8.4 \mathrm{GHz}$ with the Very Large Array (NRAO) and found the flux to be $0.30 \pm 0.04 \mathrm{mJy}$, with an excitation parameter of 1.8 , compatible with a B3 spectral type. Our flux determination is in agreement, within the errors, with previous results.

\section{IRAS 17460-3114}

Crampton (1971) classified it as an O7.5 star in a H II region; this was confirmed by Dachs et al. (1982). Gies (1987) and Mason et al. (1998) found a O8V spectral type. On the basis of its far-IR colors similar to PNe and its spectral type Parthasarathy (1993a) classified IRAS 17460-3114 as a hot post-AGB star.

Radio measurements were made by Ratag et al. (1990), who found a flux of $0.8 \mathrm{mJy}$ at $4.8 \mathrm{GHz}$ using the VLA.

We detect this source with a flux density at $8.4 \mathrm{GHz}$ of $1.29 \pm 0.05 \mathrm{mJy}$. Combining our results with data from Ratag et al. (1990), we derive a spectral index $\alpha=0.85$, consistent with an optically thick nebula, at least between 4.8 and $8.4 \mathrm{GHz}$ as we expected from a very young, and thus dense, PN.

\section{IRAS $18062+2410$}

According to the HDE catalogue its spectral type was A5 in 1940, but Downes \& Keyes (1988) classified it as a Be star. Arkhipova et al. (1999) and Partasarathy et al. (2000b) analyzed the high resolution spectrum of this star and found several permitted and forbidden emission lines: the presence of [N II] and [S II] lines indicate the presence of a low excitation nebula. Rapid photometric variability has also been detected (Arkhipova et al. 2001a) which, together the observed spectral variations, appears to indicate that the star has been evolving rapidly in the last 150 years.

\section{IRAS 18371-3159}

Preite-Martinez (1988) selected this star from the IRAS PSC as a planetary nebula candidate on the basis of its farIR colors. Parthasarathy et al. (2000a) found its spectral type to be B1Iabe, which combined with its high galactic latitude,
far-IR colors similar to PN, and Balmer line emission, makes it a post-AGB candidate.

\section{IRAS 18442-1144}

This source is very near to BD-11 4747 and possibly constitutes a binary system or is identifiable with it. Corporon \& Lagrange (1999) did not find any evidence of binariety. Parthasarathy et al. (2000a) found an A3V spectral type for BD-11 4747, but they could not observe the optical counterpart of IRAS 18442-1144 because it was probably too faint to detect. On the basis of the presence of [N II] lines in the spectrum IRAS 18442-1144 may be a post-AGB star or a low excitation PN.

We found no source at the coordinates of BD-11 4747, which is reported as the optical counterpart of IRAS 184421144. A distinct object was detected at RA $=18: 47: 04$ and Dec $=-11: 41: 12$, with a flux density of $19.21 \pm 0.06 \mathrm{mJy}$.

The radio positions are shifted by only $\Delta \alpha=3^{\prime \prime}$ and $\Delta \delta=$ $1^{\prime \prime}$ with respect to the IRAS coordinates, well inside the IRAS ellipse error $\left(30^{\prime \prime} \times 7^{\prime \prime}\right)$. We can thus conclude that the radio source is associated to IRAS 18442-1144.

If our data are combined with those from NVSS it appears that the nebula around IRAS $18442-1144$ is optically thick between 1.4 and $8.4 \mathrm{GHz}$.

\section{IRAS 19336-0400}

Downes \& Keyes (1988) found [N II] and [S II] lines in the spectrum of IRAS 19336-0400, suggesting the presence of a nebula. Parthasarathy et al. (2000a) classified it as a B1 supergiant with Balmer lines in emission, but without [O III] line, thus suggesting it is a very young and low excitation planetary nebula. IRAS 19336-0400 was detected by van de Steene \& Pottash (1995) with the WRST at $4.8 \mathrm{GHz}$ as a $10.5 \pm 1.1 \mathrm{mJy}$ radio source. The source is also present in the NVSS with a flux density at $1.4 \mathrm{GHz}$ of $8.8 \pm 0.6 \mathrm{mJy}$. We measured a $8.4 \mathrm{GHz}$ radio flux density of $9.74 \pm 0.3 \mathrm{mJy}$.

Combining our data with those from the literature we derive a quite flat radio spectrum, indicating a nebula which is optically thin between 1.4 and $8.4 \mathrm{GHz}$.

\section{IRAS 19590-1249}

Ultraviolet, optical and infrared observations of IRAS 19590-1249 (McCausland et al. 1992; Conlon et al. 1993) suggest that this B0 supergiant is in the post-AGB evolutionary stage, evolving into a low excitation planetary nebula. By comparing the atmospheric parameters of the stars with theoretical post-AGB evolutionary tracks a central mass of $0.67 M_{\odot}$ has been estimated (Conlon et al. 1993), implying that further evolution can occur on a timescale not more than hundreds of years. Photometric variability has been reported (Arkhipova et al. 2002), with a pattern similar to what has been observed in other two of our detected targets, namely IRAS $18062+2410$ and IRAS 20462+3416, which appears to be attributable to their common evolutionary status.

\section{IRAS 20462+3416}

Turner \& Drilling (1984) classified this star as a B1.5 supergiant, but, on the basis of its high galactic latitude, 
far-IR colours similar to PN, dust shell parameters and flux distribution, Parthasarathy (1993b), concluded that it was a low mass post-AGB star, with a detected cold dust shell. The optical spectrum of IRAS $20462+3416$ consists of the spectrum of a B1.5 supergiant plus the emission spectrum of a low-excitation nebula, with no [O III] lines (Parthasaraty 1993b; Smith \& Lambert 1994). Strong spectral variations were observed (Smith \& Lambert 1994; Garcia-Lario et al. 1997b), which are interpreted in terms of mass-loss episodes. Evidence of mass-loss has been reported also by Arrieta \& Torres-Peimbert (2002) from the analysis of P Cyg profiles observed in both UV and optical spectra. As in other hot postAGB star, the star exhibited rapid, irregular light variation, that can be attributed to a variable stellar wind and/or to some kind of stellar pulsations (Arkhipova et al. 2001b).

We want to stress that only few of our targets have been studied in detail. In particular, multi-epoch photometric and spectroscopic studies are available only for IRAS $06556+1623$, IRAS 17423-1755, IRAS 18062+2410, IRAS 19590-1249 and IRAS $20462+3416$. Such studies indicated a strong variability in both continum and spectral lines.

We may conclude that, besides selection criteria and the observed free-free emission, a possible common characteristic to our sources is strong spectral variability which may indicate fast evolution through episodic mass-loss events.

\section{Nebular characteristics}

Since the aim of this work is to find new objects in the very initial stage of PNe evolution, we need to check if our detected objects are indeed young PNe. We will first determine the radio characteristics of our sample and then we will check how they compare with those of young PNe as derived from previous, more extended radio surveys.

\subsection{Brightness temperature}

From measurements of the radio continuum of PNe it is possible to determine a distance-independent parameter, namely the brightness temperature $\left(T_{\mathrm{B}}\right)$, which is defined as:

$T_{\mathrm{B}}=\left(\frac{c^{2}}{2 \pi k v^{2}}\right) \frac{F_{v}}{\theta^{2}}$

where $F_{v}$ is the flux density at frequency $v$, and $\theta$ is the angular diameter of the nebula. For $v=8.4 \mathrm{GHz}$ Eq. (3) becomes:

$T_{\mathrm{B}}=25\left(\frac{\theta}{\operatorname{arcsec}}\right)^{-2} \frac{F_{8.4 \mathrm{GHz}}}{\mathrm{mJy}} \mathrm{K}$.

At the resolution of our observations 4 detected targets are point-like. In these cases we can derive only a lower limit for the brightness temperature, assuming for the sources an angular diameter $\leq 2$. For the rest of the target the angular size has been derived by a Gaussian fitting of the source in the map (see Sect. 2.2) and by performing a Gaussian deconvolution of the fitted source size.
Table 2. Summary of nebular characteristics of detected targets. Angular diameters are corrected following van Hoof (2000); The mean emission measure $(E M)$ has been calculated from the formula of Terzian \& Dickey (1973); IRE are derived following Pottasch (1984).

\begin{tabular}{lccccc}
\hline \hline IRAS ID & $\begin{array}{c}\text { Diameter } \\
{[\operatorname{arcsec}]}\end{array}$ & $\begin{array}{c}T_{\mathrm{B}} \\
{[\mathrm{K}]}\end{array}$ & $\begin{array}{c}E M \\
{\left[10^{4} \mathrm{~cm}^{-6} \mathrm{pc}\right]}\end{array}$ & IRE & $\begin{array}{c}T_{\text {dust }} \\
{[\mathrm{K}]}\end{array}$ \\
\hline $06556+1623$ & 2.1 & 2.3 & 6.3 & 194 & 181 \\
$17381-1616$ & $\leq 2.0$ & $\geq 8.9$ & $\geq 18.8$ & 31 & 218 \\
$17423-1755$ & $\leq 2.0$ & $\geq 1.6$ & $\geq 3.4$ & 2984 & 130 \\
$17460-3114$ & 1.1 & 27 & 56.6 & 248 & 204 \\
$18062+2410$ & $\leq 2.0$ & $\geq 9.1$ & $\geq 19.3$ & 106 & 253 \\
$18371-3159$ & $\leq 2.0$ & $\geq 3.9$ & $\geq 8.2$ & 187 & 187 \\
$18442-1144$ & 1.8 & 148 & 314.8 & 11 & 129 \\
$19336-0400$ & 1.5 & 108 & 229.8 & 14 & 187 \\
$19590-1249$ & 1.9 & 19.1 & 40.6 & 21 & 134 \\
$20462+3416$ & 2.2 & 2.2 & 4.6 & 186 & 114 \\
\hline
\end{tabular}

If we indicate with $\theta_{\text {syn }}$ the $F W H M$ of the point-source response of the interferometer and with $\theta_{\mathrm{G}}$ the fitted $F W H M$ size, the resultant deconvolved $F W H M$ size $(\theta)$ is:

$\theta=\sqrt{\left(\theta_{\mathrm{G}}\right)^{2}-\left(\theta_{\mathrm{syn}}\right)^{2}}$

which, for resolved sources, can be significatively smaller than the nominal resolution of the interferometer.

The obtained angular sizes were then corrected for the appropriate factor $(\gamma)$ following van Hoof (2000),

$\gamma(\beta)=\frac{0.36}{1+0.7994 \beta^{2}}+1.1106$

where $\beta$ represents the ratio between $\theta_{\mathrm{G}}$ and $\theta_{\text {syn }}$.

The results are summarized in Table 2. As the nebula expands, as a consequence of PN evolution, its surface brightness is expected to decrease. Thus $T_{\mathrm{B}}$ is usually considered an age indicator, as high $T_{\mathrm{B}}$ should characterize compact, very young PNe. $T_{\mathrm{B}}$ measured for large samples of $\mathrm{PNe}$ are in the range between 10000 and $0.1 \mathrm{~K}$ (Kwok 1990). Our results indeed indicate very low $T_{\mathrm{B}}$ values for our sample. This, however, can be still in agreement with the hypothesis of young age if one assumes that at the observing frequency the optical depth of the nebula is very small.

Among the targets of our sample there are three sources, namely IRAS 17460-3114, IRAS 18442-1144 and IRAS 19336-0400, whose radio spectrum can be inferred on the basis of data from the literature and the results presented in this paper (see Sect. 3). It can be argued that the first two sources appear to be optically thick, at least between 20 and $3.6 \mathrm{~cm}$. Unfortunately, the available spectral information is too poor and a two-point spectrum, in one case obtained very far apart in frequency, is not sufficient to draw any conclusion on the spectral index. 


\subsection{Emission measure}

Another physical parameter whose high value should characterize a young PN is the electron density which is expected to decrease as the nebula ages and expands.

The mean emission measure $(\langle E M\rangle)$, as derived from radio observations, is an averaged property of the ionized nebula and, therefore, may better represent the overall density structure of PNe.

If the source is optically thin we can use the observed radio flux density plus its angular size to derive the mean emission measure as follows (Terzian \& Dickey 1973):

$\langle E M\rangle=\frac{\int_{\Omega} E M \mathrm{~d} \Omega}{\Omega}=\frac{5.3 \times 10^{5} F_{8.4 \mathrm{GHz}}}{\theta^{2}}$

where $F_{8.4 \mathrm{GHz}}$ is the measured radio flux density, in mJy, at $8.4 \mathrm{GHz}$, and $\theta$, in arcsec, the angular dimension of the radio emitting region at that frequency. The mean emission measure is in $\mathrm{cm}^{-6} \mathrm{pc}$.

Young PNe should have emission measures of the order of $10^{6}-10^{8} \mathrm{~cm}^{-6} \mathrm{pc}$ (Terzian \& Dickey 1973; Kwok et al. 1981) and, as reported in Table 2, our sample, in general, appears to have a more diluted nebula.

\subsection{Infrared excess}

A young planetary nebula is still surrounded by the remnant of the dusty envelope of its AGB progenitor and emits most of its energy in the far infrared as thermal radiation from heated dust grains. The infrared excess (IRE) is defined as the ratio of the observed total far infrared flux $\left(F_{\mathrm{IR}}\right)$ over the expected total infrared flux. On the hypothesis that the far infrared flux is due to thermal emission from dusty grains heated by Ly $\alpha$ photons, this ratio is unity. In young and compact planetary nebulae dust heating by direct absorption of starlight is important, and therefore this ratio can be much larger than unity. For this reason the infrared excess is often used as an age indicator for PNe. Since the expected total infrared flux can be expressed in terms of optically thin radio flux density, we may derive IRE from the formula given by Pottasch (1984). In particular, as the nebulae associated to our targets are compact, the infrared excess is derived in the high density approximation, i.e.:

$\mathrm{IRE}=1.07 \frac{F_{\mathrm{IR}}}{F_{8.4 \mathrm{GHz}}}$

where $F_{\mathrm{IR}}$, in $10^{-14} \mathrm{~W} \mathrm{~m}^{-2}$, is the total infrared flux. For consistent comparison with other similar studies, $F_{\text {IR }}$ has been derived by fitting a Planck curve over the IRAS bands, namely from 12 to $100 \mu \mathrm{m}$ (Aaquist \& Kwok 1990). Our sample is characterized by IRE values much higher than unity. As a byproduct of this method we also derive the dust color temperature. A summary of the nebular characteristics of our sample is reported in Table 2.

\section{Comparison with another sample}

In order to check if our sample consists of young PNe, we will compare the radio and infrared properties of the targets

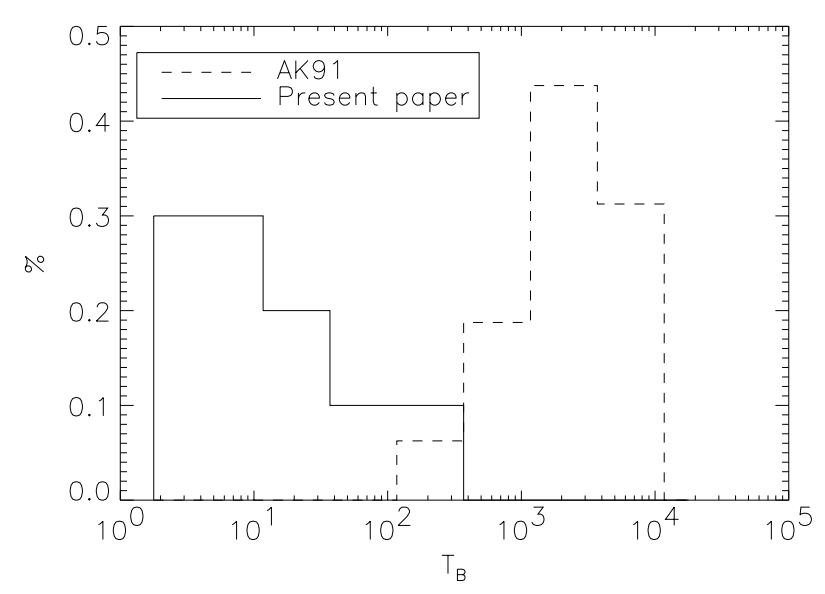

Fig. 1. Distribution of brightness temperature $\left(T_{\mathrm{B}}\right)$ for the AK91 sample (dashed line) and the sample of the present paper (full line). $T_{\mathrm{B}}$ for AK91 were calculated from Eq. (3) and using radio measurements obtained at $15 \mathrm{GHz}$. Four of our targets have only lower limits for $T_{\mathrm{B}}$.

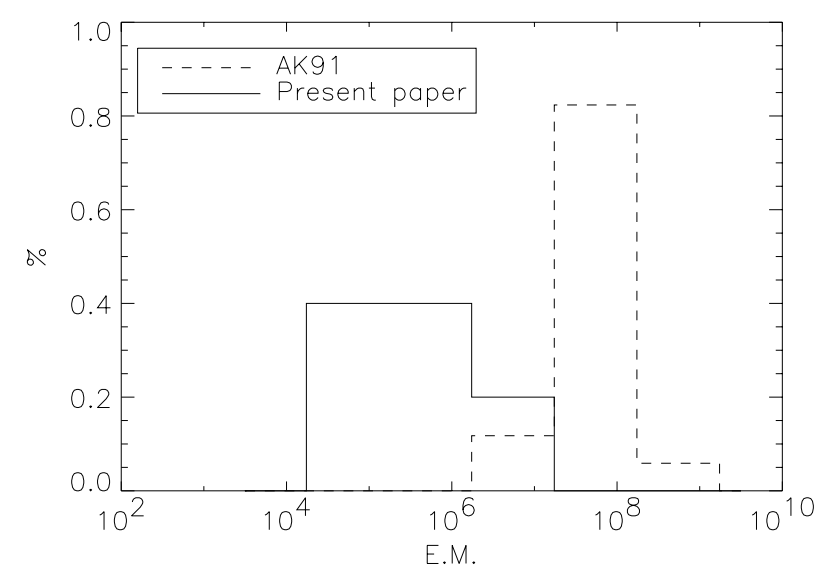

Fig. 2. Distribution of mean emission measure (EM) of the AK91 sample (dashed line) and of the present sample (full line). Four of our targets have only lower limits for $E M$.

belonging to our sample with results obtained in similar studies. In particular, in the following, we will consider the work of Aaquist \& Kwok (1991, AK91) carried out with the VLA at $15 \mathrm{GHz}$ on a sample of young PNe selected on the basis of their compact radio morphology. The targets of AK91 were also observed at $5 \mathrm{GHz}$, but we prefer to compare our results with those obtained at $15 \mathrm{GHz}$ since in both cases optical depth effects should not be important.

All the selected targets in AK91 have high brightness temperatures, infrared excess (IRE) much higher than unity and dust temperature higher than the typical value observed in more evolved nebulae, which is of the order of $100 \mathrm{~K}$ (Pottasch et al. 1984). All these properties are consistent with the hypothesis that the sample consists of very young PNe.

In order to compare the physical properties of the nebulae belonging to AK91 with our sample we plot in Figs. 1-3 the brightness temperature $\left(T_{\mathrm{B}}\right)$, the emission measure $(E M)$ and the infrared excess (IRE) of both samples. Those quantities were re-calculated from the published radio measurements using the same formulas as for our sample. 


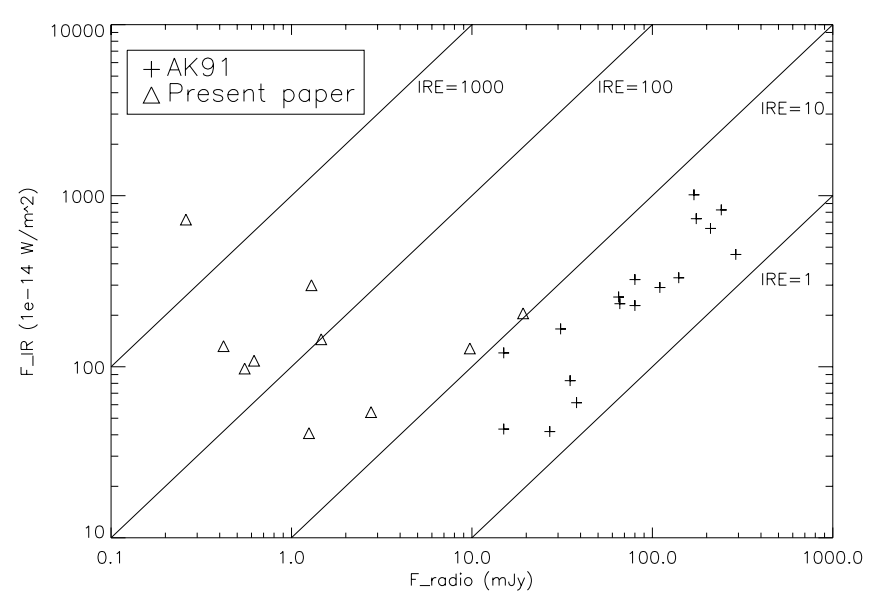

Fig. 3. The radio flux density versus the far infrared flux for the present sample (empty triangle) and for the AK91 sample (plus signs).

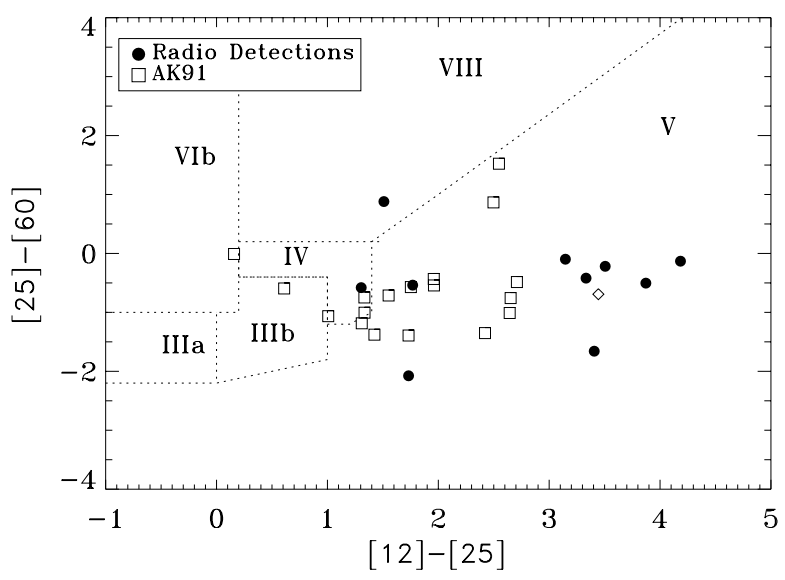

Fig. 4. IR color-color diagram of detected sources (filled dots) sources. Open squares are data from AK91. The position in the colorcolor diagram of the very young PN SAO 244567 is also marked as an empty diamond. Regions in the IRAS two-colors diagram for different kinds of source are also indicated (following van der Veen \& Habing 1988).

It is evident that for the AK91 sample $T_{\mathrm{B}}$ and $E M$ are systematically higher than for our sample, and this seems to indicate that our sample indeed consists of more evolved PNe.

On the contrary, the infrared excess of our sample, which has values systematically higher than those reported by AK91, indicates a particularly young sample of PNe. This apparent contradiction is further complicated by the fact that the infrared properties of both samples are quite similar, as is evident from an inspection of the IRAS color-color diagram (Fig. 4), where sources belonging to different samples occupy the same region of the diagram. This region is also shared with SAO244567, the youngest known PN, whose evolution appears to be quite rapid, since it has become ionized only within the past 20 years (Parthasarathy et al. 1993).

In the diagram the infrared colors are defined as usual (van der Veen \& Habing 1988):

$[12]-[25]=-2.5 \log \frac{F_{12}}{F_{25}}$

where $F_{12}$ and $F_{25}$ are the fluxes measured at 12 and $25 \mu$.

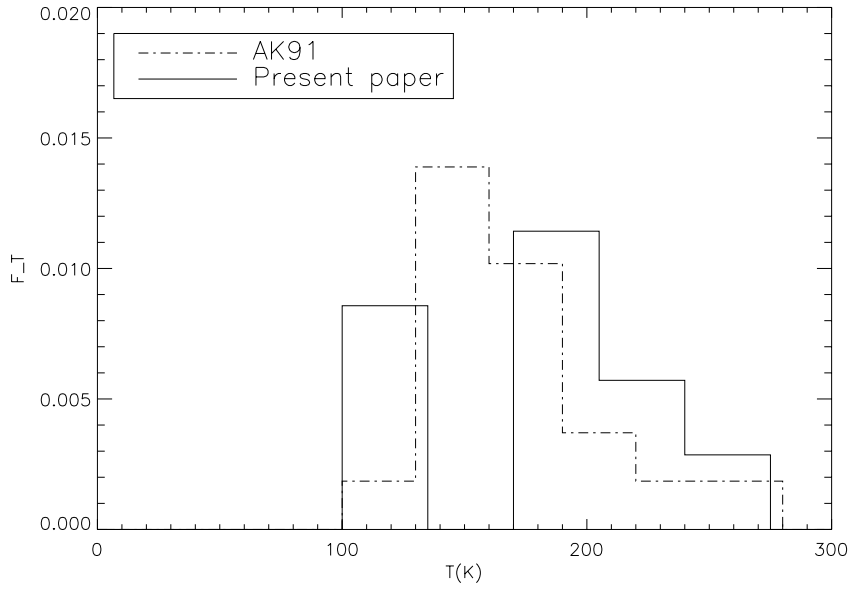

Fig. 5. Distribution of dust temperature of the AK91 sample (dashed line) and of the present sample (full line).

Moreover, dust temperatures of both samples have quite similar distributions (Fig. 5), strengthening the conclusion that both samples have similar dust characteristics.

Concerning the possible causes of lower $T_{\mathrm{B}}$ and $E M$ of our sample we may consider whether this could be related to a systematic effect due to the different spatial resolution used in the two surveys, as both $T_{\mathrm{B}}$ and $E M$ depend on the angular source size $\left(\propto \theta^{-2}\right)$.

A factor 20 in spatial resolution yields a two orders of magnitude difference in both $T_{\mathrm{B}}$ and EM, forcing the two distributions to be more similar than what is shown in Figs. 1 and 2. However, only 4 out of 10 detected sources were not resolved and thus without a good angular size estimate. Therefore, while this effect cannot be ruled out, at least for these 4 sources, we will consider other possibilities which may explain our results.

Volk \& Kwok (1985) studied the dynamical evolution of a nebula in the framework of the interacting wind model. They considered all the possible factors that can affect the dynamics of the ejecta, including radiative cooling, variation of mass-loss of the stellar wind as well as evolution of the central object.

The authors also reproduced, in the context of their dynamical model, the time evolution of the radio flux density, assuming that all the radio emission arises only from the ionized part of the shell.

The evolution of the radio flux density with the age of the nebula is outlined in Fig. 6, adapted from Fig. 15 of Volk \& Kwok (1985), where three distinct phases of radio emission have been indicated.

In particular, during the first phase, when the radio emitting region is bounded by the ionized front, the radio flux increases with the radius of the ionized nebula, which is growing with the age of the nebula. This is strictly related to the rate of Lyman continuum photons which increases with the central star evolution (Kwok 1985).

In this context we may try to solve the apparent inconsistency between the typical values of $T_{\mathrm{B}}$ and $E M$, as derived for our sample, which indicate a more evolved sample, and the IRE values, which, on the contrary, appear to be consistent with a population of very young PNe. 


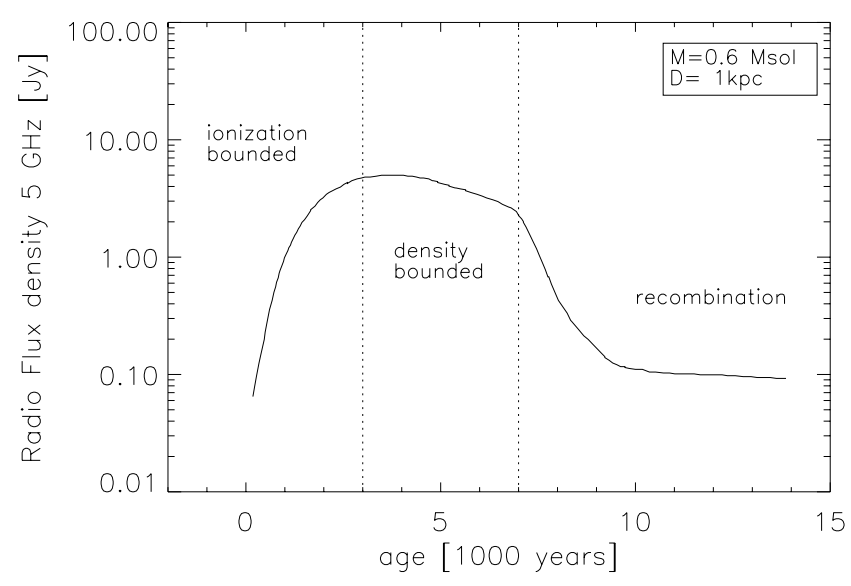

Fig. 6. Evolution of the radio flux density in the framework of the dynamical model of Volk \& Kwok (1985). Adapted from Volk \& Kwok (1985). The curve is obtained assuming a nebula at a distance of $1 \mathrm{kpc}$, with a core-mass of $0.6 M_{\odot}$ in the hypothesis of a stellar wind with high mass-loss $\left(10^{-4} M_{\odot} \mathrm{yr}^{-1} \leq \dot{M} \leq 10^{-5} M_{\odot} \mathrm{yr}^{-1}\right)$

If we consider the functional dependence of $T_{\mathrm{B}}, E M$ and IRE on the radio flux density $\left(F_{\text {radio }}\right)$, the first two being proportional to $F_{\text {radio }}$ and IRE to $F_{\text {radio }}^{-1}$, our contradictory result can be explained if we assume that, on average, our sample is characterized by a very low radio flux compared to what was measured in the AK91 sample.

We thus conclude that the present sample is, indeed, very young and that the ionization in the circumstellar nebula has just started.

Therefore, only a small fraction of the nebula is ionized and in the evolutionary scheme, reproduced in Fig. 6, they occupy the very initial portion of the ionization bounded phase, corresponding to a very low value of radio flux density. On the contrary, the AK91 sample, even if consisting of young PNe, occupies a more advanced portion of the same evolutionary phase, corresponding to a much higher radio flux.

To further test our hypothesis, we calculate the ionized mass of the nebula from the observed radio flux, following Pottasch (1984):

$M_{i}=5.11 \times 10^{-5}\left(F_{8.4} \theta d^{5}\right)^{1 / 2}$

where it is assumed that the nebula has a filling factor $\epsilon=0.6$ and that $10 \%$ of its mass consists of helium atoms. In the formula $F_{8.4}$ is in mJy, $\theta$ is in arcsec, $d$ in kpc and $M_{\mathrm{i}}$ in $M_{\odot}$. If we assume a standard distance of $1 \mathrm{kpc}$ for the sources detected in our survey, we obtain values for the ionized mass of the nebula in the range $6 \times 10^{-5} \leq \frac{M_{\mathrm{i}}}{M_{\odot}} \leq 5.4 \times 10^{-4}$, which are much lower that the typical value of $0.2 M_{\odot}$ obtained for more evolved nebulae (Pottasch 1984). If we assume that all the nebulae are at a standard distance of $10 \mathrm{kpc}$, we obtain values $2 \times 10^{-2} \leq \frac{M_{i}}{M_{\odot}} \leq 17 \times 10^{-2}$. These are to be considered upper limits.

A radio nebula was recently detected around SAO244567 (Umana et al. 2004) and preliminary results were presented by Trigilio et al. (2003). A $T_{\mathrm{B}}=700 \mathrm{~K}$ and a $E M=1.5 \times$ $10^{7} \mathrm{~cm}^{-6}$ pc place SAO244567 between our sample and AK91 and this implies, in the framework of our hypothesis, that this young PN possesses a radio nebula slightly more evolved than those in our sample.

\section{Summary}

The formation and early evolution of Planetary Nebulae is an unclear phase of stellar evolution. It is still not well understood how the circumstellar envelopes, which form during the AGB phase, transform themselves into the complex morphologies often observed in evolved PNe (Sahai 2002; Kwok et al. 2002). In order to understand the origin of such morphologies, it is very important to clarify at what stage of PN evolution the nebula becomes asymmetric or bipolar. Moreover, recently some objects have been identified whose evolution towards the PN phase occurs on timescales twenty times shorter than what is foreseen by current evolutionary models (Parthasarathy 2000). In this context it appears to be quite important to find objects in transition towards the PN phase, which can provide strong constraints on determining which process shapes the nebula and how this influences the nebular evolution.

In order to improve our knowledge in this field we have observed a sample of 16 hot post-AGB stars, selected on the basis of their optical and infrared properties. A common feature of our targets is an infrared excess, signature of mass-loss that occurred during the AGB phase. The main result of the present work is the detection of free-free radio emission in ten of the observed sources. This indicates that ionization has already started in their circumstellar material and that the sources can be considered to be in a very early stage of Planetary Nebulae evolution. Therefore, the detected sources constitute a unique sample to be studied for shedding light on this quite obscure evolutionary phase.

To further test our results, we made a comparison with another sample of PNe assumed to be young. We preferred the sample studied by Aaquist \& Kwok (1991), as both samples were observed at high frequencies and any possible opacity effect can be neglected. By comparing the nebular characteristics of both samples, as derived from radio measurements, we conclude that our sample comprises extremely young PNe. The apparent inconsistency between radio and infrared properties can be explained by assuming that in our nebulae the ionization has just started, while in the nebulae from AK91 the ionization structure is more evolved. This translates into a difference in the radio emission, which is, on average, less intense for our sample. This result is strengthened by the fact that we derive values of ionized mass of the nebula which are much lower than those usually derived for more evolved nebulae.

Successive multi-frequency and high spatial resolution radio observations will allow us to fully characterize the radio properties of these new objects, providing important clues to better understand the formation and shaping of PN in the early stages of their evolution.

Acknowledgements. This research has made use of the SIMBAD database, operated at CDS, Strasbourg, France. 


\section{References}

Aaquist, O. B., \& Kwok, S. 1990, A\&AS, 84, 229

Aaquist, O. B., \& Kwok, S. 1991, ApJ, 378, 599 (AK91)

Altenhoff, W. J., Braes, L. L .E., Olnon, F. M., \& Wendker, H. J. 1976, A\&A, 46, 11

Arkhipova, V. P., \& Ipatov, A. P. 1982, SvA Lett., 8, 298

Arkhipova, V. P., Ikonnikova, N. P., Noskova, R. I., et al. 1999, Astron. Lett., 25, 25

Arkhipova, V. P., Klochkova, V. G., \& Sokol, G. V. 2001a, Astron. Lett., 27, 99

Arkhipova, V. P., Ikonnikova, N. P., Noskova, R. I., et al. 2001b, Astron. Lett., 27, 719

Arkhipova, V. P., Ikonnikova, N. P., Noskova, R. I., \& Komissarova, G. V. 2002, Astron. Lett., 28, 257

Arrieta, A., \& Torres-Peimbert, S. 2002, Rev. Mex. Astron. Astrofis., 12,154

Bains, I., Gledhill, T. M., Yates, J. A., \& Richards, A. M. S. 2003, MNRAS, 338, 287

Bobrowsky, M., Zijlstra, A. A., Grebel, E. K., et al. 1995, ApJ, 446, L89

Borkowski, K. J., \& Harrington, J. P. 2001, ApJ, 550, 778

Cahn, J. H., \& Kaler, J. B. 1971, ApJS, 22, 319

Ciatti, F., D'Odorico, S., \& Mammano, A. 1974, A\&A, 34, 181

Condon, J. J., Cotton, W. D., Greisen, E. W., et al. 1998 AJ, 115, 1693

Conlon, E. S., Dufton, P. L., McCausland, R. J. H., \& Keenan, F. P. 1993, ApJ, 408, 599

Corporon, P., \& Lagrange, A.-M. 1999, A\&AS, 136, 429

Crampton, D. 1971, AJ, 76, 260

Dachs, J., Kaiser, D., Sherwood, W. A., \& Nikolov, A. 1982, A\&AS, 50,261

Downes, R. A., \& Keyes, C. D. 1988, AJ, 96, 777

Garcia-Lario, P., Manchado, A., Pych, W., \& Pottasch, S. R. 1997a, A\&AS, 126, 479

Garcia-Lario, P., Parthasarathy, M., de Martino, D., et al. 1997b, A\&A, 326,1103

Gauba, G., Parthasarathy, M., Kumar, B., Yadav, R. K. S., \& Sagar, R. 2003, A\&A, 404, 305

Gies, D. R. 1987, ApJS, 64, 545

Houk, N. 1982, Catalog of two-dimensional spectral types for the HD stars, Vol. 3, Michigan Spectral Survey, Ann Arbor, Dep. Astron., Univ. Michigan

Ivezic, Z., Nenkova, M., \& Elitzur, M. 1999, User Manual for DUSTY, University of Kentucky Internal Report

Jaschek, C., Andrillat, Y., \& Jaschek, M. 1996, A\&AS, 117, 281

Klutz, M., \& Swings, J. P. 1977, A\&A, 56, 143

Knapp, G. R., Bowers, P. F., Young, K., \& Phillips, T. G. 1995, ApJ, 455,293
Kwok, S. 1994, PASP, 106, 344

Kwok, S. 1990, MNRAS, 244, 179

Kwok, S., Purton, C. R., \& Keenan, D. W 1981, ApJ, 250, 232

Kwok, S., Su, K. Y. L., \& Sahai, R. 2002, A\&AS, 197, 603

Lee, C. F., \& Sahai, R. 2003, ApJ, 586, 319

Mason, B. D., Gies, D. R., Hartkopf, W. I., et al. 1998, AJ, 115, 821

McCausland, F. J. H., Conlon, E. S., Dufton, P. L., \& Keenan, F. P. 1992, ApJ, 394, 298

Parthasarathy, M. 1993a, Luminous High-Latitude Stars, ASP Conf. Ser., 45,173

Parthasarathy, M. 1993b, ApJ, 414, L109

Parthasarathy, M. 2000, Bull. Astro. Soc. India, 28, 217

Parthasarathy, M., \& Pottasch, S. R. 1989, A\&A, 225, 521

Parthasarathy, M., Garcia-Lario, P., Pottasch, S. R., et al. 1993, A\&A, 267, L19

Parthasarathy, M., Vijapurkar, J., \& Drilling, J. S. 2000a, A\&AS, 145, 269

Parthasarathy, M., Garcia-Lario, P., Sivarani, T., Manchado, A., \& Sanz Fernandez de Cordoba, L. 2000b, A\&A, 357, 241

Pottasch, S. R. 1984, Planetary nebulae - a study of late stages of stellar evolution (Dordrecht: D. Reidel Publishiing Co.)

Pottasch, S. R., Baud, B., Beintema, D., et al. 1984, A\&A, 138, 10

Pottasch, S. R., Olling, R., Bignell, C., \& Zijlstra, A. A. 1988, A\&A, 205, 248

Preite-Martinez, A. 1988, A\&AS, 76, 317

Ratag, M. A., Pottash, S. R., Zijlstra, A. A., \& Menzies, J. 1990, A\&A, 233,181

Riera, A., Garcia-Lario, P., Manchado, A., Pottasch, S. R., \& Raga, A. C. 1995, A\&A, 302, 137

Riera, A., Garcia-Lario, P., Manchado, A., Bobrowsky, M., \& Estalella, R. 2003, A\&A, 401, 1039

Sanchez Contreras, C., \& Sahai, R. 2001, ApJ, 553, L173

Sahai, R. 2002, Rev. Mex. Astron. Astrofis., 13, 133

Sahai, R., \& Trauger, J. T. 1998, AJ, 116, 1357

Sistla, G., \& Seung, H. 1975, A\&A, 44, 477

Smith, V. V., \& Lambert, D. L. 1994, ApJ, 424, L123

Terzian, Y., \& Dickey, J. 1973, AJ, 78, 875

Terzian, Y. 1993, Planetary Nebulae, ed. R. Weinberger, \& A. Acker (Dordrecht: Kluwer), IAU 155, 109

Trigilio, C. , Umana, G., \& Cerrigone, L. 2003 [arXiv:astro-ph/0310288]

Turner, D. G., \& Drilling, J. S. 1984, Publ. Astr. Soc. Pacific, 96, 292

Umana, G., Trigilio, C., \& Cerrigone, L. 2004, in preparation

van de Steene, G. C. M., \& Pottasch, S. R. 1993, A\&A, 274, 895

van de Steene, G. C. M., \& Pottasch, S. R. 1995, A\&A, 299, 238

van der Veen, W. E. C. J., \& Habing, H. J. 1988, A\&A, 194, 125

van Hoof, P. A. M. 2000, MNRAS, 314, 99

Volk, K., \& Kwok, S. 1985, A\&A, 153, 79 